\title{
Advancements in Integrated Micro-XRF in the SEM
}

\author{
K.C. Witherspoon,* B.J. Cross**, R.D. Lamb, ${ }^{*}$ and P.-O. Sjoman* \\ * IXRF Systems, Inc., 15715 Brookford Drive, Houston, TX 77059 \\ ** CrossRoads Scientific, P.O. Box 1823, El Granada, CA 94018
}

Over the last few years, small x-ray tubes have been modified for mounting on Scanning Electron Microscopes. There have been two main types: (a) low-power miniature tubes mounted re-entrantly within the SEM [1], and (b) higher-power tubes with integrated x-ray optics to produce smaller beam spots at the sample with intensities still high enough for routine analytical work [1,2]. This addition allows samples to be analyzed both by X-Ray Fluorescence (XRF), and by the electron beam (SEM-EDS), as illustrated with the two spectra in FIG. 1.

Both techniques can be used independently or together by taking sequential e-beam and x-ray excited spectra. Quantitative analysis using this combined approach was first demonstrated at the IMC16 conference in Sapporo [3]. This approach uses the advantage of e-beam excitation for lighter elements below $2.0 \mathrm{keV}$, and the more-efficient XRF excitation for $\mathrm{x}$-ray lines above 2.0 $\mathrm{keV}$. Micro-XRF with X-Y stage scanning can be used to collect X-ray elemental maps similar to those collected with e-beams, except that the stage is scanned instead of the beam. This Micro-XRF mapping method has been proposed for some time [e.g. 4], and was first commercially demonstrated in 1986 [5]. In addition it is possible to collect e-beam and x-ray excited maps simultaneously, by scanning the X-Y stage, for combined qualitative X-ray elemental mapping (FIG. 2).

Currently a 40 micron x-ray beam spot size is available inside the SEM. Recently, a 10 micron beam has been demonstrated, with count rates that can exceed $2000 \mathrm{cps}$ on steel. In the future we expect even smaller excitation areas, with "useful" x-ray count rates. To create a smaller spot, the polycapillary optic needs to be more tightly focussed. This means that the Focal (i.e., working) Distance (FD) of the XRF source must be shorter. For example, with a 40 micron spot, an FD of 11 $\mathrm{mm}$ is typical. With a 10 micron excitation spot, an FD of about 4-5 mm will be required. This makes the integration of the x-ray beam much more challenging (FIG. 4).

In the future, "brighter" x-ray sources will be integrated offering more x-ray flux. It is now possible to use primary filters (thin foils) in front of the x-ray source. Using an automated filter wheel, this allows in situ tuning of the x-ray source spectrum [e.g. 4], with improved elemental detection limits. An automated filter wheel between the x-ray source and sample provides comparable capabilities to those in a benchtop XRF. FIG. 5 shows a comparison of unfiltered and filtered spectra, showing how the overall "shape" can be varied to optimize sensitivities and peak-to-background ratios.

Most SEM's have either motorized stages, or can be retrofitted with one (FIG. 6). These stages are conventionally used for sample positioning in the SEM, rather than mapping. Of course the stages must be scanned to collect XRF maps and so endurance and reliability is at a premium because of the higher work loads. Retrofitting an SEM with an X-Y stage requires careful attention to the inner components and space within the chamber. Other considerations include speed, accuracy, and backlash, as well as endurance. Because heat dissipation in vacuo is poor, heat transfer is also critical for most stages, especially if run continuously. 
References

[1] B.J. Cross and K.C. Witherspoon, Microsc. Microanal. 10 (Suppl. 2), (2004) 104.

[2] M. Procop and V.-D. Hodoroaba, Microsc. Microanal. 13 (Suppl. 2), (2007) 1424.

[3] B.J. Cross and K.C. Witherspoon, Proc. IMC16, Sapporo, (2006) 879.

[4] E.P. Bertin, Principles and Practice of $X$-ray Spectrometric Analysis, $2^{\text {nd }}$ Ed., Plenum, New York, (1975).

[5] D.C. Wherry and B.J. Cross, Kevex Analyst, (Aug. 1986) 8.

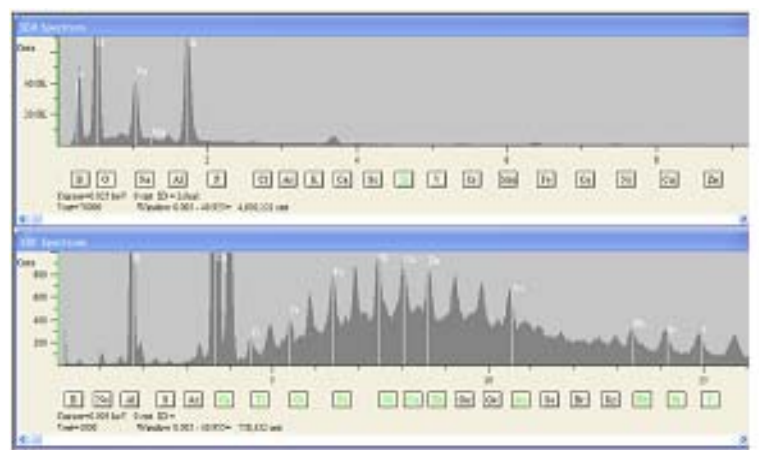

Fig. 1. SEM-EDS spectrum (top) and Micro-XRF (bottom) of SRM 610.

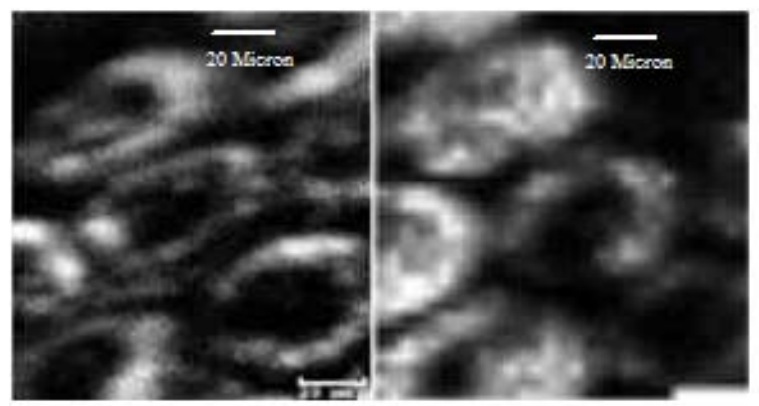

Fig. 3. Improved resolution from the 10 vs. 40 micron beam.

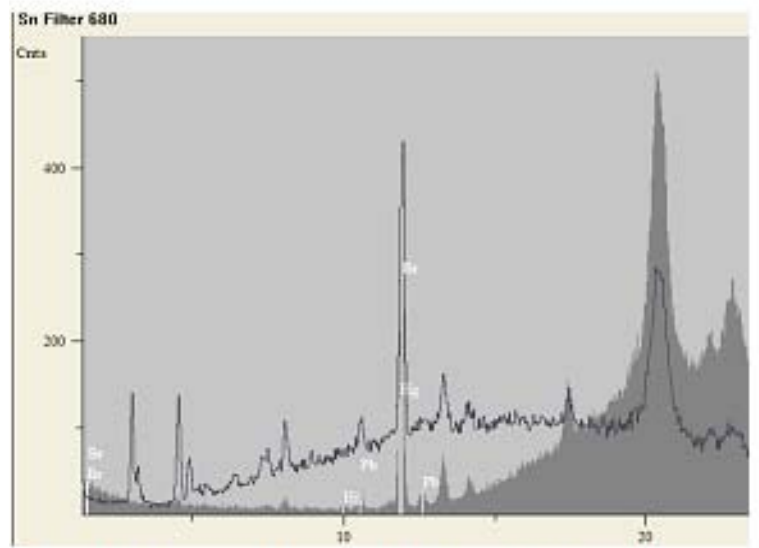

Fig. 5. Comparison of filtered and unfiltered tube spectra.

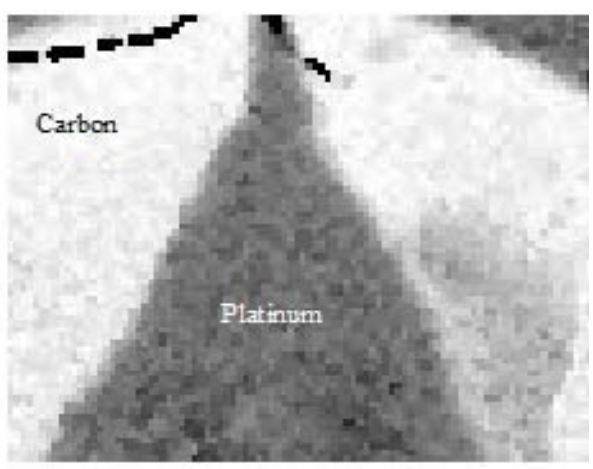

Fig. 2. Combined x-ray maps from simultaneous XRF and SEM-EDS acquisition.

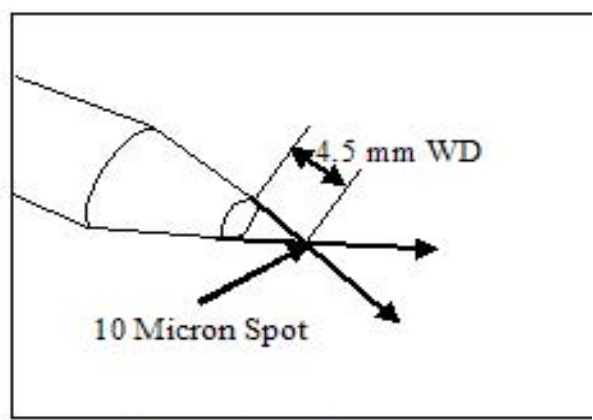

Fig. 4. Close focal distance required for a 10-micron spot polycapillary optic.

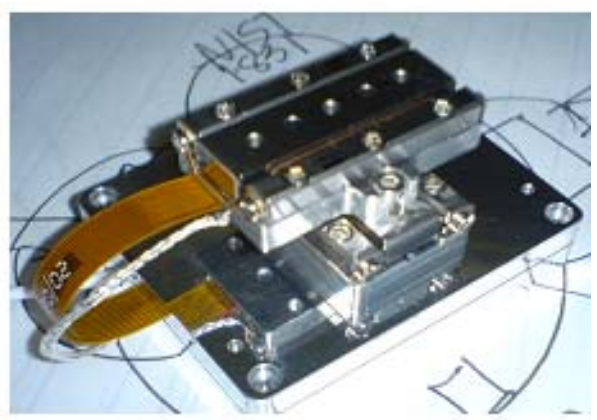

Fig. 6. X-Y stage for retrofitting in SEM. 